\title{
Xeroderma pigmentosum: Squamous cell carcinoma infiltrating and disfiguring facial, in a girl of 3 years and a half
}

\section{Laouali Salissou', Moussa Doulla1, Mahamadou Zaki Harouna², Illé Salha ${ }^{3}$, Nasser Timmi ${ }^{3}$, Amadou Dan Sono Abdou ${ }^{3}$, Hassan Moumouni ${ }^{4}$, Rachid Sani ${ }^{5}$, Hassane Nouhou ${ }^{2}$}

${ }^{1}$ Department of Dermatology, National Hospital Niamey, Niamey, Niger, ${ }^{2}$ Laboratory of pathological anatomy, Faculty of Health Sciences, Niamey, Niger, ${ }^{3}$ Department of ORL National Hospital of Niamey, Niamey, Niger, ${ }^{4}$ Laboratory of Cytology and Genetic, Faculty of Health Sciences, Niamey, Niger, ${ }^{5}$ Department of Surgery, National Hospital Niamey, Niamey, Niger

Corresponding author: Dr. Laouali Salissou, E-mail: danmata@yahoo.com

\begin{abstract}
Most of serious complications observed during the development of Xeroderma pigmentosum (XP) are cancerous. These include skin, eyes, tongue, nervous system, etc. We report the case of a 3 l/2-year-old girl with squamous cell carcinoma infiltrating and disfiguring the face with rapid onset of death.
\end{abstract}

Key words: Xeroderma pigmentosum; Infiltrating epidermoid carcinoma; Niger 


\title{
Xeroderma pigmentosum: Carcinome spinocellulaire infiltrant et délabrant du visage, chez une fillette de 3 ans et demi
}

\author{
Laouali Salissou', Moussa Doulla', Mahamadou Zaki Harouna², Illé Salha ${ }^{3}$, \\ Nasser Timmi ${ }^{3}$, Amadou Dan Sono Abdou ${ }^{3}$, Hassan Moumouni ${ }^{4}$, Rachid Sani ${ }^{5}$, \\ Hassane Nouhou²
}

${ }^{1}$ Department of Dermatology, National Hospital Niamey, Niamey, Niger, ${ }^{2}$ Laboratory of pathological anatomy, Faculty of Health Sciences, Niamey, Niger, ${ }^{3}$ Department of ORL National Hospital of Niamey, Niamey, Niger, ${ }^{4}$ Laboratory of Cytology and Genetic, Faculty of Health Sciences, Niamey, Niger, ${ }^{5}$ Department of Surgery, National Hospital Niamey, Niamey, Niger

Corresponding author: Dr. Laouali Salissou, E-mail: danmata@yahoo.com

\begin{abstract}
RÉSUMÉ
La plupart des complications graves observées au cours de l'évolution du Xeroderma pigmentosum (XP) sont de nature cancéreuse. Celles-ci concernent notamment la peau, mais également les yeux, la langue, le système nerveux, etc. Nous rapportons le cas d'une fillette âgée de 3 ans et demie ayant présenté un carcinome épidermoïde infiltrant et délabrant du visage avec la survenue rapide de décès.
\end{abstract}

Mots clés: Xeroderma pigmentosum; Carcinome épidermoïde infiltrant, Niger

\section{INTRODUCTION}

Le Xeroderma Pigmentosum (XP), est une maladie rare de transmission autosomique récessive, caractérisée par une sensibilité pathologique aux ultras violets, liée à un déficit des systèmes enzymatiques de réparation de l'ADN. Son évolution est le plus souvent compliquée par l'apparition précoce de cancers cutanés et extra cutanés [1]. Nous rapportons un cas, caractérisé par la survenue très précoce de carcinome spinocellulaire infiltrant du visage, avec délabrement quasi-totale de la face et entrainant le décès rapide de la patiente à l'âge de trois ans et demi.

\section{OBSERVATION}

Il s'est agit d'une patiente âgée de 3 ans et demi, troisième d'une fratrie de 5 enfants et reçue en consultation dermatologique le 04 mai 2015. Il n'y a une notion de consanguinité, les deux grandpères étant des frères. Les premiers signes à type de photophobie et de xerodermie ont été rapportés à l'âge de 6 mois. A l'âge de 3 ans apparaissait une tumeur nodulaire de $2 \mathrm{~cm}$ à la partie moyenne du nez qui est devenue par la suite ulcérocroûteuse et impétiginisée (Fig. 1). L'examen systémique était normal sans trouble neurologique, sans taie ou hémorragie conjonctivale. Pendant ses 3 consultations à 5 jours d'intervalle, au service de Dermatologie, la patiente a bénéficié d'un bilan (Radiographie thoracique, Numération formule sanguine, VS, urée, créatininémie, CRP, l'ionogramme sanguin et les transaminases) qui était normal, des soins locaux et une antibiothérapie générale (Erythromycine). Par la suite elle était perdue de vue pendant 3 mois. C'était le 05 Août 2015 (3 mois plus tard) que nous avons appris son hospitalisation au service d'ORL de l'Hôpital National de Niamey. A l'examen ce jour, on décrivait une grande ulcération

\footnotetext{
How to cite this article: Salissou L, Doulla M, Harouna MZ, Salha I, Timmi N, Sono Abdou AD, Moumouni H, Sani R, Nouhou H. Xeroderma pigmentosum: Carcinome spinocellulaire infiltrant et délabrant du visage, chez une fillette de 3 ans et demi. Our Dermatol Online. 2017;8(Suppl. 1):28-31.

Submission: 05.05.2017; Acceptance: 22.07.2017

DOI: 10.7241 /ourd.2017s.7
} 
allant de la lèvre supérieure aux yeux délabrant tout au passage avec obstruction des fosses nasales (Fig. 2). Lexamen anatomopathologique d'une pièce de biopsie révélait un carcinome spinocellulaire infiltrant bien différencié (Fig. 3). Une chimiothérapie a été initiée associant la cisplatine et la 5 fluorouracile pour un total de 3 séances à 3 semaines d'intervalle. La progression de l'ulcération s'est accélérée malgré ce traitement (Fig. 4). Létat général s'est rapidement dégradé et la patiente décéda en Octobre 2015. On ne notait aucun cas familial.

\section{DISCUSSION}

Le diagnostic du XP est clinique et ne pose habituellement pas de problème devant les signes classiques inauguraux dont la photophobie et la xérodermie [2]. L'incidence de Xeroderma Pigmentosum est de 1 cas sur 250000 habitants [3] et semble plus

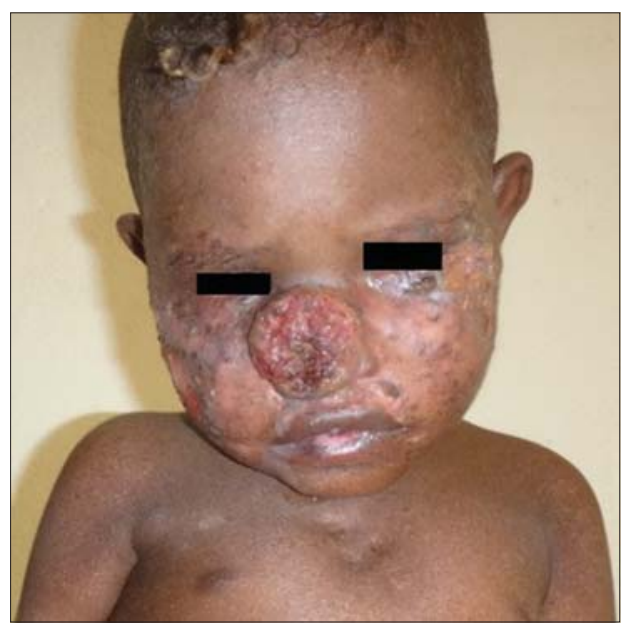

Figure 1: Lésion bourgeonnante parcourant tout le nez.

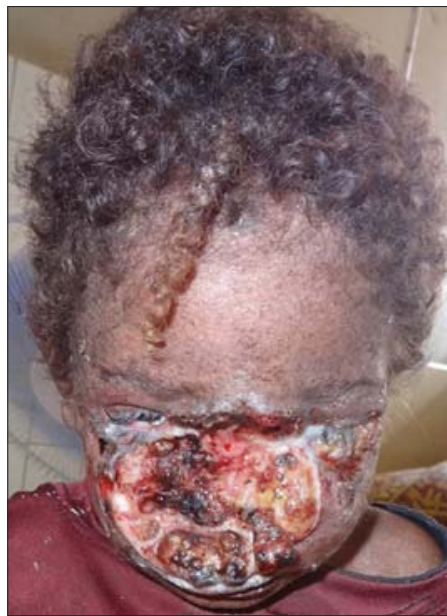

Figure 2: Ulcération délabrante de la lévre supérieure aux yeux, avec obstruction des fosses nasales. importante au japon et au Mahgreb avec 1 cas pour 100000 habitants [4]. Sa survenue, malgré rare sur peau noire est cependant caractérisée par l'apparition précoce et fréquente des tumeurs cancéreuses notamment le carcinome spinocellulaire $[1,5,6]$. Chez notre patiente l'examen anatomopathologique a révélé un carcinome spinocellulaire infiltrant bien différencié. Contrairement à certaines séries $[1,5,7]$, l'atteinte oculaire a été faite par délabrement progressif par cancer, à partir du nez. Certains auteurs ont rapporté l'atteinte linguale $[2,5]$ qui fait très souvent suite à l'atteinte cutanée. Notre patiente était indemne d'autres atteintes carcinomateuses telles que le mélanome [8], ou la leucémie lymphoblastique aiguë [9] en dehors de celle oculaire. Le xeroderma pigmentosum est décrit dans 7 groupes de complémentarité (XPA à XPG) et 1 groupe variant XPV [10,11]. Malgré l'impossibilité de réaliser un examen génétique, l'absence de déficit neurologique dans le groupe XPC $[11,12]$ classe cette patiente dans ce dernier.

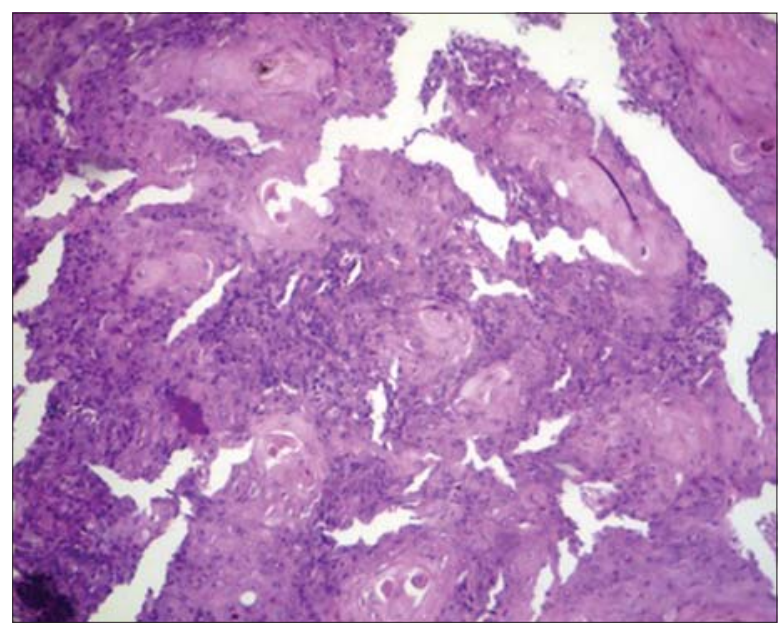

Figure 3: Carcinome spinocellulaire kératinisant.

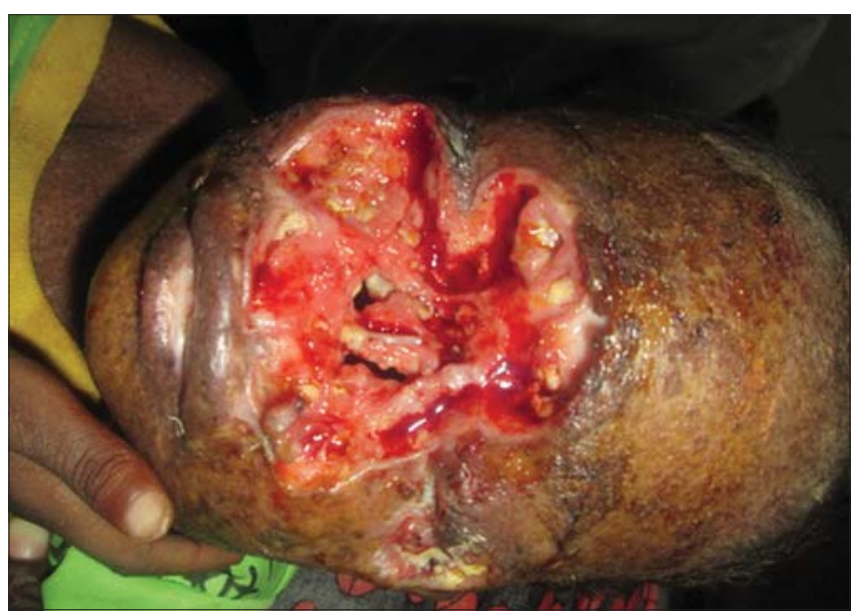

Figure 4: Ulcération délabrant l'oeil droit, avec atteinte débutante de l'oeil gauche. 
La prise en charge du XP est toujours très coûteuse dans les pays développés que dans ceux en voie de développement [13] comme le Niger, et se limite à la photoprotection, les soins locaux et l'antibiothérapie en cas de surinfection. Les soins plus spécialisés dont la chirurgie et la chimiothérapie sont effectués en concertation pluridisciplinaire [12]. Tout récemment la prise en charge par chimiothérapie est à ses premiers pas à l'HNN. Le traitement chez cette patiente a associé deux antimitotiques ( 5 fluorouracile et cisplatine). Cette prise en charge par ces antimitotiques a trouvé ses limites étant donné le caractère subintrant de la survenue des cancers chez le patient XP [14]. Certains traitements sont préventifs pour la survenue de cancers cutanés et consistent à l'utilisation de l'isotrétinoïne [15]. Lévolution se fait pratiquement toujours vers un décès précoce [9]. Le décès était survenu à l'âge de 3 ans et demi chez notre patiente, comme il est rapporté dans la littérature que les formes XPC sont résistantes à la chimiothérapie [16].

\section{CONCLUSION}

Malgré sa survenue rare chez le sujet de phénotype foncé, le pronostic de XP est sombre avec l'apparition précoce de cancers dont les plus fréquents sont d'origine cutanée. L'environnement très ensoleillé dans un pays comme le Niger, est un facteur très favorable dans la survenue précoce et l'évolution rapide de ces cancers. Linterdiction de mariage consanguin doit être imposée dès lors qu'il existe des antécédents familiaux de XP.

\section{RÉFÉRENCES}

1. Kraemer KH, Lee MM, Scotto J. Xeroderma Pigmentosum: Cutaneous, ocular, and neurologic abnormalities in 830 published cases. Arch Dermatol. 1987;123:241-0.

2. Salissou L, Moumouni H, Sani R, Nouhou H. Xeroderma pigmentosum: première observation au Niger. Ann Univsers A M Niamey. 2013;XV-A:1-8.
3. Alain S, Georges R, ClaudineB-B, JoelleB, Yves D. Le XP: caractéristiques cliniques, génétiques et cellulaires, développement d'un test anténatal. Med/Scien. 1988;4:608-17.

4. Shetty S, Girish BS, Ballal R, Harish SP, Makannavar P, Alva V. Xeroderma pigmentosum with multiple cutaneous malignancies- A case report and review of literatue. NUJHS. 2013;3:76-8.

5. Pathy S, Naik KK, Bhasker S, Manghera PS, Malhotra H. Squamous Cell Carcinoma of Face With Xeroderma Pigmentosum: A Case Report. Indian J Med and Pediatric Oncoloy. 2005;26:47-9.

6. Bouadjar B, Belkacem FA, Daya-Grosjean L, Larbaoui LS, Ferhat R, Cherid MC, et al. Xeroderma pigmentosum, Etude de 40 malades algériens. Ann. Dermatol. Venereol. 1996;123:305-6.

7. Ahmed H, Hassan R Y, Pindiga U. Xeroderma in three consecutive siblings of a Nigerian family: Observations of oculocutaneous manifestations in black African children. Br J Ophtalmol. 2001;85:110-1.

8. Fazaa B, Zghal M, Zeglaoui F, Goucha S, Mokhtar I, Kharfi M, et al. Mélanome et Xeroderma pigmentosum: 12cas. Ann Dermatol Venereol. 2001;128:503-6.

9. Dieng MT, Niang SO, Dangou JM, Ndiaye B. Xeroderma pigmentosum: report of 6 cases in Dakar, Senegal. Bull Cancer. 2001;88:199-202.

10. Pradhan E, Padhye SB, Malla OK, Karki KJD. Case of xeroderma pigmentosum with well differentiated squamous cell carcinoma in the eye. Khatmandu Univers Med Journ. 2003;1:278-84.

11. Sethi M, Lehmann A.R, Fassihi H. Xeroderma pigmentosum: A multidisciplinary approach. Europ Med Journ. 2013;54-63.

12. Cleaver JE, Feeney L, Tang JY, Tuttle P. Xeroderma pigmentosum group $\mathrm{C}$ in an isolated region of Guatemala. J Invest Dermatol. 2007;127:493-6.

13. Hari Kishan KY, Syeda J. Xeroderma Pigmentosum: A Bane in developing country - Brief report. Our Dermatol Online. 2014;4:395-7.

14. Moussais M, Benchikhi H, Boukind EH, Sqalli S, Mouaki N, Kadiri F, et al. Tumeurs cutanées au cours de xeroderma pigmentosum au Maroc. Ann Dermatol Venerol. 2004;131:29-33.

15. Kaemer KH, DIGiovannaJJ, Moshell AN,Tarone RE, Peck GL. Preventio skin cancer in xeroderma pigmentosum with the use of oral isotretinoin. N Engl J Med. 1988;319:926-9.

16. Jerbi M, Ben Rekaya M, Nouali C, Jones M, Tounsi H, Messaoud O, et al. Profils cliniques et génétiques de patients atteints de xeroderma pigmentosum forme C: à propos de 64 patients tunisiens. Ann Dermatol Venerol. 2015;JDP 2015, P202.

Copyright by Laouali Salissou, et al. This is an open-access article distributed under the terms of the Creative Commons Attribution License, which permits unrestricted use, distribution, and reproduction in any medium, provided the original author and source are credited.

Source of Support: Nil, Conflict of Interest: None declared. 